Y. KAKIHARA

KODAI MATH. J.

6 (1983), 289-300

\title{
ON A HILBERT MODULE OVER AN OPERATOR ALGEBRA AND ITS APPLICATION TO HARMONIC ANALYSIS
}

\author{
BY YÛICHIRÔ KAKIHARA
}

\section{Introduction.}

We study a left $A$-module with an $A$-valued inner product where $A$ is an operator algebra. Such a space has been investigated by many authors: Kaplansky [7], Saworotnow [14], Paschke [12], Rieffel [13], Ozawa [11], Itoh [5], Kakihara and Terasaki [6] and others.

Let $A$ be a von Neumann algebra. Then a Hilbert $A$-module is defined to be a left $A$-module with an $A$-valued inner product respecting the module action, called a Gramian, which is complete with respect to (w.r.t.) the norm induced from the Gramian. Our main object is harmonic analysis on a topological group in the Hilbert $A$-module context. Especially, a Stone type and a Bochner type theorems are formulated and proved.

Basic definitions of a Hilbert $A$-module are given in section 2. In section 3 , $A$-valued positive definite kernels are considered in connection with reproducing kernel Hilbert $A$-modules which are analogous to Aronszajn's reproducing kernel Hilbert spaces [1]. Section 4 deals with Gramian unitary representations of a topological group and Gramian *-representations of a $L^{1}$-group algebra on a Hilbert $A$-module. Results stated in sections 3 and 4 hold when $A$ is a (unital) $C^{*}$-algebra. In section 5 , we prove our main result which is a Stone type theorem for a continuous, in an appropriate sense, Gramian unitary representation of a locally compact abelian group. As a corollary we give a proof to a Bochner type theorem for a weakly continuous $A$-valued positive definite function. Section 6 is devoted to Hilbert $A$-module valued processes over a locally compact abelian group. Such a formulation of processes is closely related to Banach space valued stochastic processes (cf. Cobanjan and Weron [2], Weron [19] and Miamee [8]).

Acknowledgments: The author would like to express his hearty thanks to Professor $H$. Umegaki for his valuable suggestions and encouragement in the course of preparing this paper.

\section{Hilbert $A$-modules.}

Throughout this paper $A$ stands for a von Neumann algebra with the norm Received June 4, 1982 
$\|\cdot\|$. We denote the action of $A$ on a left $A$-module $X$ by $(a, x) \rightarrow a \cdot x, a \in A$, $x \in X$. We assume that all such modules treated below have a vector space structure compatible with that of $A$ in the sense that $\alpha(a \cdot x)=(\alpha a) \cdot x=a \cdot(\alpha x)$ for $x \in X, a \in A$ and a complex number $\alpha$.

2.1. Definition. A (left) pre-Hilbert A-module is a left $A$-module for which there is a map $[\cdot, \cdot]: X \times X \rightarrow A$ such that for $x, y, z \in X$ and $a \in A$ (1) $[x, x]$ $\geqq 0$, and $[x, x]=0$ iff $x=0$; (2) $[x+y, z]=[x, z]+[y, z]$; (3) $[a \cdot x, y]=a[x, y]$; (4) $[x, y]^{*}=[y, x]$. The map $[\cdot, \cdot]$ is called a Gramian on $X$. We sometimes denote it explicitly by $[\cdot, \cdot]_{X}$.

If $X$ is a right $A$-module, then we can define (right) pre-Hilbert $A$-module structure for $X$ in a similar manner as above except that the condition (3) is replaced by $\left(3^{\prime}\right)[x \cdot a, y]=[x, y] a$. Since there is no essential difference between right and left $A$-modules, we restrict our attention to left $A$-modules.

In a pre-Hilbert $A$-module $X$ define $\|x\|_{X}=\|[x, x]\|^{1 / 2}, x \in X$. Then by $[12$, 2.3 Proposition], $\|\cdot\|_{X}$ becomes a norm on $X$ and we have for $x, y \in X$ and $a \in A$

$$
\|a \cdot x\|_{X} \leqq\|a\| \cdot\|x\|_{X}, \quad\|[x, y]\| \leqq\|x\|_{X} \cdot\|y\|_{X} .
$$

2.2. Definition A pre-Hilbert $A$-module $X$ which is complete w.r.t. the norm $\|\cdot\|_{X}$ is called a Hilbert A-module.

Examples of (right) Hilbert $A$-modules are seen in [12] where $A$ is a $C^{*}$ algebra.

2.3. Definition. Let $X$ be a Hilbert $A$-module. We define the Gramian orthogonal complement of a subset $Y$ of $X$ by $Y^{\#}=\{x \in X ;[x, y]=0, y \in Y\}$. A subset $Y$ is called a submodule if it is a left $A$-module and is closed w.r.t. $\|\cdot\|_{X}$. In this case $Y$ is itself a Hilbert $A$-module. Denote by $\subseteq(Y)$ the submodule generated by a subset $Y$. It is seen that for each subset $Y$ its Gramian orthogonal complement $Y^{\#}$ is a submodule and the relation $\widetilde{S}(Y) \subset\left(Y^{\#}\right)^{\#}$ holds.

2.4. Definition. Let $X$ and $Y$ be two Hilbert $A$-modules with Gramians $[\cdot, \cdot]_{X}$ and $[\cdot, \cdot]_{Y}$, respectively. $B(X, Y)$ denotes the Banach space of all bounded linear operators from $X$ into $Y . \mathfrak{A}(X, Y)$ denotes the set of all operators $S \in B(X, Y)$ for which there is an operator $T \in B(Y, X)$ such that $[S x, y]_{Y}$ $=[x, T y]_{X}, x \in X, y \in Y$. It is seen that $T$ is unique if it exists, so that we denote it by $S^{*}$ and call it the Gramian adjont of $S$. An operator $U \in B(X, Y)$ is said to be Gramian unitary if it is onto and satisfies that $\left[U x, U x^{\prime}\right]_{Y}=\left[x, x^{\prime}\right]_{X}$, $x, x^{\prime} \in X$. It can be seen that each Gramian unitary operator $U \in B(X, Y)$ belongs to $\mathfrak{A}(X, Y)$ and satisfies $U^{*} U=I_{X}$, the identity operator on $X$. We write $B(X)$ $=B(X, X)$ and $\mathfrak{A}(X)=\mathfrak{U}(X, X)$. An operator $P \in B(X)$ is called a Gramian projection if $P \in \mathfrak{A}(X)$ and $P^{2}=P^{*}=P$. Two Hilbert $A$-modules $X$ and $Y$ are said to be isomorphic, in symbols $X \cong Y$, if there is a Gramian unitary operator in 
$\mathfrak{A}(X, Y)$.

For $a \in A$ define $\pi(a)$ by $\pi(a) x=a \cdot x, x \in X, X$ being a Hilbert $A$-module. Then, by $(2.1), \pi(a) \in B(X)$. A kind of functionals on a Hilbert $A$-module is defined in the following (cf. $[7,12,14]$ ).

2.5. Definition. Let $X$ be a Hilbert $A$-module. Denote by $X^{*}$ the set of all bounded module maps $\tau: X \rightarrow A$. That is, $\tau$ satisfies $\tau(a \cdot x+b \cdot y)=a \tau(x)+$ $b \tau(y), x, y \in X, a, b \in A$, and there is some $\alpha>0$ such that $\|\tau(x)\| \leqq \alpha\|x\|_{X}, x \in X$. Each $x \in X$ gives rise to a map $\hat{x} \in X^{*}$ defined by $\hat{x}(y)=[y, x], y \in X . \quad X$ is said to be self-dual if $X^{*}=\hat{X}(=\{\hat{x} ; x \in X\})$.

2.6. Remark ([12]). Let $X$ be a Hilbert $A$-module. Then $X^{*}$ becomes a self-dual Hilbert $A$-module in which $X$ can be embedded as a submodule. Moreover, each operator in $\mathfrak{A}(X)$ can be uniquely extended to an operator in $\mathfrak{A}\left(X^{*}\right)$. If $X$ is self-dual, then we have $\mathfrak{A}(X)=\{S \in B(X) ; S \pi(a)=\pi(a) S, a \in A\}$. That is, $\mathfrak{A}(X)$ consists of all bounded module maps from $X$ into itself. Furthermore, there is a collection $\left\{p_{\imath} ; i \in \mathfrak{S}\right\}$ of (not necessarily distinct) nonzero projections in $A$ such that $X \cong \operatorname{UDS}\left\{A p_{\imath} ; \imath \in \Im\right\}$, the ultraweak direct sum of self-dual Hilbert $A$-modules $A p_{\imath}, i \in \mathfrak{J}$. For each $\imath \in \mathfrak{S}$ the Gramian on $A p_{\imath}$ is defined by $\left[a p_{2}, b p_{2}\right]_{2}=a p_{2} b^{*}, a, b \in A$. As a consequence of this decomposition, for any self-dual submodule $Y$ of $X$, we have that $X=Y \oplus Y^{\#}$, the direct sum, and that there is a Gramian projection of $X$ onto $Y$.

\section{Positive definite kernels.}

We consider $A$-valued positive definite kernels on $\Omega \times \Omega, \Omega$ being a set, and construct reproducing kernel Hilbert $A$-modules.

3.1. Definition. An $A$-valued function $\Gamma$ on $\Omega \times \Omega$ is called a positive definite kernel $(P D K)$ if for every finite $\left\{\omega_{1}, \cdots, \omega_{n}\right\} \subset \Omega$ and $\left\{a_{1}, \cdots, a_{n}\right\} \subset A$ it holds that $\sum_{i, j} a_{i} \Gamma\left(\omega_{i}, \omega_{j}\right) a_{\jmath}^{*} \geqq 0$. Every PDK $\Gamma$ on $\Omega \times \Omega$ satisfies that $\Gamma\left(\omega, \omega^{\prime}\right)$ $=\Gamma\left(\omega^{\prime}, \omega\right)^{*}, \omega, \omega^{\prime} \in \Omega$.

For each $A$-valued PDK $\Gamma$ on $\Omega \times \Omega$ we can associate a Hilbert $A$-module $\Omega \otimes_{\Gamma} A$ by the method similar to that of Umegaki [17]. To this end, let $F(\Omega ; A)$ be the set of all $A$-valued functions on $\Omega$ with finite supports. For $f, g \in F(\Omega ; A)$ and $a \in A$ define $(a \cdot f)(\cdot)=a f(\cdot),[f, g]_{\Gamma}=\sum_{\omega, \omega^{\prime}} f(\omega) \Gamma\left(\omega, \omega^{\prime}\right) g\left(\omega^{\prime}\right)^{*}$ and $\|f\|_{\Gamma}=$ $\left\|[f, f]_{\Gamma}\right\|^{1 / 2}$. Then $[\cdot, \cdot]_{\Gamma}$ satisfies conditions of a Gramian except that $[f, f]_{I^{\prime}}$ $=0$ implies $f=0$. Put $N_{\Gamma}=\left\{f \in F(\Omega ; A) ;[f, f]_{\Gamma}=0\right\}$ and let $\Omega \otimes_{\Gamma} A$ be the completion of the quotient space $F(\Omega ; A) / N_{\Gamma}$ w.r.t. the norm $\|\cdot\|_{\Gamma}$. Then $\Omega \otimes_{\Gamma} A$ is a Hilbert $A$-module. Moreover, it is closely related to the reproducing kernel Hilbert $A$-module of $\Gamma$ defined below. 
3.2. Definition. Let $\Gamma$ be an $A$-valued PDK on $\Omega \times \Omega$ and $X$ be a Hilbert $A$-module consisting of $A$-valued functions on $\Omega$. Then $X$ is said to be the reproducing kernel $(R K)$ Hilbert A-module of $\Gamma$ if

(1) for each $\omega \in \Omega, \Gamma(\omega, \cdot) \in X$;

(2) for each $\omega \in \Omega$ and $x \in X, x(\omega)=[x(\cdot), \Gamma(\omega, \cdot)]$.

The PDK $\Gamma$ is called the reproducing kernel $(R K)$ for $X$.

3.3. Proposition. For each $A$-valued PDK $\Gamma$ on $\Omega \times \Omega$ there is a unique, up to isomorphism, Hilbert A-module $X_{\Gamma}$ admitting $\Gamma$ as a $R K$. Moreover, the relation $X_{\Gamma} \cong \Omega \otimes_{\Gamma}$ A holds.

Proof. The proof mimics that of $[9,2.5$. Lemma $]$ and we only give the outline. Let $X_{0}$ be the set of all $A$-valued functions on $\Omega$ of the form

$$
x(\cdot)=\sum_{\imath=1}^{n} a_{i} \Gamma\left(\omega_{i}, \cdot\right), \quad a_{i} \in A, \omega_{i} \in \Omega, 1 \leqq \imath \leqq n
$$

with $n$ finite. Define for $x(\cdot)=\sum a_{i} \Gamma\left(\omega_{i}, \cdot\right), y(\cdot)=\sum b_{j} \Gamma\left(\omega_{j}^{\prime}, \cdot\right) \in X_{0}$ and $a \in A$

$$
(a \cdot x)(\cdot)=\sum_{\imath} a a_{i} \Gamma\left(\omega_{i}, \cdot\right), \quad[x, y]_{0}=\sum_{j, j} a_{i} \Gamma\left(\omega_{i}, \omega_{\jmath}^{\prime}\right) b_{j}^{*} .
$$

Then $X_{0}$ becomes a pre-Hilbert $A$-module with an action and a Gramian defined as above. Moreover, for $x \in X_{0}$ and $\omega \in \Omega$ the reproducing property $x(\omega)=$ $[x(\cdot), \Gamma(\omega, \cdot)]_{0}$ holds. Hence we have $\|x(\omega)\| \leqq\|x\|_{0} \cdot\|\Gamma(\omega, \cdot)\|_{0}$ where $\|y\|_{0}=$ $\left\|[y, y]_{0}\right\|^{1 / 2}, y \in X_{0}$.

Let $\left\{x_{n}\right\}$ be a Cauchy sequence in $X_{0}$ w.r.t. the norm $\|\cdot\|_{0}$. It follows from the above inequality that for every $\omega \in \Omega$ there exists some $x(\omega) \in A$ such that $\left\|x_{n}(\omega)-x(\omega)\right\| \rightarrow 0$. Denote by $X_{\Gamma}$ the set of all $A$-valued functions $x$ on $\Omega$ obtained in this way. For $x, y \in X_{\Gamma}$ define $[x, y]=\lim _{n \rightarrow \infty}\left[x_{n}, y_{n}\right]_{0}$ where $\left\{x_{n}\right\}$ and $\left\{y_{n}\right\}$ are Cauchy sequences in $X_{0}$ determining $x$ and $y$, respectively. Then we can check that $X_{\Gamma}$ is actually a Hilbert $A$-module with the Gramian $[\cdot, \cdot]$. Furthermore, the reproducing property of $\Gamma$ can also be checked and, therefore, $\Gamma$ is a RK for $X_{\Gamma}$. The uniqueness of $X_{\Gamma}$ and the isomorphism $X_{\Gamma} \cong \Omega \otimes_{\Gamma} A$ are readily verified.

\section{Gramian unitary reresentations and Gramian *-representations.}

We first consider Gramian unitary representations of a topological group on a Hilbert $A$-module and their relation to $A$-valued positive definite functions on the group.

4.1. Definition. Let $G$ be a topological group and $X$ be a Hilbert $A$-module. An $A$-valued function $\Gamma$ on $G$ is said to be positive definite $(P D)$ if for every finite $\left\{a_{1}, \cdots, a_{n}\right\} \subset A$ and $\left\{s_{1}, \cdots, s_{n}\right\} \subset G$ it holds that $\sum_{\imath, j} a_{i} \Gamma\left(s_{\jmath}^{-1} s_{\imath}\right) a_{\jmath}^{*} \geqq 0$. Putting $\tilde{\Gamma}(s, t)=\Gamma\left(t^{-1} s\right), s, t \in G, \Gamma$ is $P D$ iff $\tilde{\Gamma}$ is a PDK on $G \times G . \quad \Gamma$ is said to be 
continuous if it is norm continuous on $G$. A Gramian unitary representation $(G U R)$ of $G$ on $X$ is a homomorphism $s \rightarrow U(s)$ from $G$ into $\mathfrak{A}(X)$ for which $U(s)$ is Gramian unitary for every $s \in G$. A GUR $s \rightarrow U(s)$ is said to be continuous if for every $x \in X$ the function $s \rightarrow U(s) x$ is norm continuous on $G$. A vector $x_{0} \in X$ is said to be cyclic for a GUR $s \rightarrow U(s)$ if $\subseteq\left\{U(s) x_{0} ; s \in G\right\}=X$.

Then we can prove the following.

4.2. Proposition. Let $G$ be a topological group and $\Gamma: G \rightarrow A$ be $P D$. Then there exist a Hilbert A-module $X, a$ GUR $s \rightarrow U(s)$ of $G$ on $X$ and a cyclic vector $x_{0} \in X$ such that $\Gamma(s)=\left[U(s) x_{0}, x_{0}\right], s \in G$. It also holds

$$
\|\Gamma(s)\| \leqq\|\Gamma(e)\|, \quad\|\Gamma(s)-\Gamma(t)\|^{2} \leqq 2\left\|\Gamma(e)-\Gamma\left(s^{-1} t\right)\right\| \cdot\|\Gamma(e)\|
$$

for $s, t \in G$ where $e$ is the identity of $G$. Furthermore, $\Gamma$ is continuous if and only if so is $s \rightarrow U(s)$.

Proof. Put $\tilde{\Gamma}(s, t)=\Gamma\left(t^{-1} s\right), s, t \in G$ and let $X$ be the $R K$ Hilbert $A$-module of $\tilde{\Gamma}$ with the Gramian $[\cdot, \cdot]$ (cf. 3.3. Proposition). Then we have $\Gamma(s)=\tilde{\Gamma}(s, e)$ $=[\tilde{\Gamma}(s, \cdot), \tilde{\Gamma}(e, \cdot)], s \in G$. Let $X_{0}$ be the set of all $A$-valued functions on $G$ of the form $\sum_{\imath=1}^{n} a_{\imath} \Gamma\left(s_{\imath}, \cdot\right), a_{i} \in A, s_{i} \in G, 1 \leqq \imath \leqq n$ with $n$ finite. For $s \in G$ define $U(s)$ on $X_{0}$ by $U(s) \sum a_{i} \Gamma\left(s_{\imath}, \cdot\right)=\sum a_{i} \Gamma\left(s s_{\imath}, \cdot\right)$. Then it is easy to see that for $x, y \in X_{0}$ the equality $[U(s) x, U(s) y]=[x, y]$ holds. Hence $U(s)$ can be uniquely extended to a Gramian unitary operator on $X$ since $X_{0}$ is dense in $X$. Thus $s \rightarrow U(s)$ is a GUR of $G$ on $X$. Putting $x_{0}=\tilde{\Gamma}(e, \cdot) \in X$, it is readily seen that $x_{0}$ is a cyclic vector for $s \rightarrow U(s)$ and that the equality $\Gamma(s)=\left[U(s) x_{0}, x_{0}\right]$ holds for $s \in G$. Two inequalities in (4.1) follow from this equality as in the case of scalar valued PD functions (cf. [18]). The last assertion is not hard to check.

In the remainder of this section let $G$ be a locally compact group with a left Haar measure $d s$ and consider the space $L^{1}\left(G ; Z_{A}\right)$ of all $Z_{A^{-}}$-valued Bochner integrable functions on $G$ w.r.t. $d s$ where $Z_{A}$ is the center of $A$, i.e., $Z_{A}=$ $\{a \in A ; a b=b a, b \in A\} . \quad L^{1}\left(G ; Z_{A}\right)$ is a Banach *-algebra whose multiplication, involution and norm are respectively defined by $(f g)(t)=\int_{G} f(s) g\left(s^{-1} t\right) d s, f^{*}(t)=$ $\Delta(t)^{-1} f\left(t^{-1}\right)^{*}$ and $\|f\|_{1}=\int_{G}\|f(s)\| d s$ for each $f, g \in L^{1}\left(G ; Z_{A}\right)$ and $t \in G$ where $\Delta$ is the modular function of $G$. Define $(a \cdot f)(\cdot)=a f(\cdot), a \in A, f \in L^{1}\left(G ; Z_{A}\right)$ and denote by $\mathfrak{L}^{1}\left(G ; Z_{A}\right)$ the left $A$-module generated by $L^{1}\left(G ; Z_{A}\right)$. Now we consider Gramian *-representations of $L^{1}\left(G ; Z_{A}\right)$ on a Hilbert $A$-module in connection with GURs of $G$.

4.3. Definition. Let $X$ be a Hilbert $A$-module. Then a map $f \rightarrow T(f)$ from $\mathfrak{L}^{1}\left(G ; Z_{A}\right)$ into $B(X)$ is called a Gramian *-representation $\left(G^{*} R\right)$ of $L^{1}\left(G ; Z_{A}\right)$ on 
$X$ if the restriction of $T$ to $L^{1}\left(G ; Z_{A}\right)$ is $\mathfrak{A}(X)$-valued and if $T(a \cdot f+b \cdot g)=$ $\pi(a) T(f)+\pi(b) T(g), T\left(f^{*}\right)=T(f)^{*}$ and $T(f g)=T(f) T(g)$ for each $f, g \in L^{1}\left(G ; Z_{A}\right)$ and $a, b \in A$ where $\pi(a) x=a \cdot x, x \in X$. A G*R $f \rightarrow T(f)$ is said to be nondegenerate if $\mathrm{S}\left\{T(f) x ; f \in L^{1}\left(G ; Z_{A}\right), x \in X\right\}=X$.

Given a continuous GUR $s \rightarrow U(s)$ of $G$ on a Hilbert $A$-module $X$, define $T(f)$ for $f \in L^{1}\left(G ; Z_{A}\right)$ by

$$
T(f) x=\int_{G} U(s)(f(s) \cdot x) d s, \quad x \in X,
$$

where the right hand side is a well-defined Bochner integral. If $X$ is self-dual, then we can show that $f \rightarrow T(f)$ is a $\mathrm{G}^{*} \mathrm{R}$ of $L^{1}\left(G ; Z_{A}\right)$ on $X$.

Let $\mathfrak{V}_{G}$ be the Borel $\sigma$-algebra of $G$ and $M\left(G ; Z_{A}\right)$ be the set of all $Z_{A^{-}}$ valued countably additive $(\mathrm{CA})$ measures, in the norm, on $\mathfrak{B}_{G}$ of bounded variations. For $\mu, \nu \in M\left(G ; Z_{A}\right)$ and $a, b \in A$ define $(a \cdot \mu+b \cdot \nu)(B)=a \mu(B)+b \nu(B)$, $\mu^{*}(B)=\mu\left(B^{-1}\right)^{*}$ and $(\mu \nu)(B)=\mu \times \nu\left(B^{\prime}\right)\left(B^{\prime}=\{(s, t) ; s t \in B\}\right)$ for $B \in \mathfrak{V}_{G}$, and $\|\mu\|=$ the total variation of $\mu$. Then $M\left(G ; Z_{A}\right)$ becomes a Banach *-algebra. $\mathfrak{M}\left(G ; Z_{A}\right)$ denotes the left $A$-module generated by $M\left(G ; Z_{A}\right)$. By a Gramian *-representation of $M\left(G ; Z_{A}\right)$ on a Hilbert $A$-module $X$ we mean a map $\mu \rightarrow T(\mu)$ from $\mathfrak{M}\left(G ; Z_{A}\right)$ into $B(X)$ whose restriction to $M\left(G ; Z_{A}\right)$ is $\mathfrak{A}(X)$-valued and which satisfies that $T(a \cdot \mu+b \cdot \nu)=\pi(a) T(\mu)+\pi(b) T(\nu), T\left(\mu^{*}\right)=T(\mu)^{*}$ and $T(\mu \nu)=T(\mu) T(\nu)$ for $\mu, \nu \in M\left(G ; Z_{A}\right)$ and $a, b \in A . L^{1}\left(G ; Z_{A}\right)$ is a Banach *-subalgebra of $M\left(G ; Z_{A}\right)$ by identifying $f \in L^{1}\left(G ; Z_{A}\right)$ with $f(s) d s \in M\left(G ; Z_{A}\right)$. By similar proofs of $[3$, 13.3.1. and 13.3.4. Propositions] we can show the following.

4.4. Proposition. Let $X$ be a self-dual Hilbert A-module. Given a continuous $G U R s \rightarrow U(s)$ of $G$ on $X$, define for $\mu \in M\left(G ; Z_{A}\right)$

$$
T(\mu) x=\int_{G} U(s) \pi(\mu(d s)) x, \quad x \in X .
$$

Then $T(\mu)$ is a well-defined operator on $X$ and $\mu \rightarrow T(\mu)$ is a $G^{*} R$ of $M\left(G ; Z_{A}\right)$ on $X$ whose restriction to $L^{1}\left(G ; Z_{A}\right)$ is nondegenerate.

If $f \rightarrow T(f)$ is a nondegenerate $G^{*} R$ of $L^{1}\left(G ; Z_{A}\right)$ on $X$, then there is a unique continuous GUR $s \rightarrow U(s)$ of $G$ on $X$ such that (4.2) holds.

\section{A Stone type and a Bochner type theorems.}

In this section we assume that $G$ is a locally compact abelian group. Denote by $A_{*}$ the predual of $A$ and by $A_{*}^{+}$its positive part. For a Hilbert $A$-module $X$ we define the Gramian $\sigma$-weak topology on $\mathfrak{A}(X)$ (or $B(X)$ ) to be the topology determined by the family of seminorms

$$
S \in \mathfrak{U}(X) \text { (or } B(X)) \rightarrow|\rho([S x, y])|, \quad x, y \in X, \rho \in A_{*}^{+} .
$$

We prove a Stone type spectral representation theorem for a Gramian $\sigma$-weakly 
continuous GUR of $G$ on some self-dual Hilbert $A$-module. As a consequence we give a proof to a Bochner type integral representation theorem of an $A$ valued weakly continuous PD function on $G$. For the scalar valued case we refer to Nakamura and Umegaki [10] and Umegaki [18].

Before we proceed we need some preparations. Let $\mathfrak{B}_{\hat{G}}$ be the Borel $\sigma$-algebra of the dual group $\hat{G}$ of $G$ and $X$ be a Hilbert $A$-module.

5.1. Definition. A map $P: \mathfrak{B}_{\hat{G}} \rightarrow \mathfrak{A}(X)$ is called a Graman spectral measure on $\hat{G}$ if $P$ is Gramian projection valued and if, for each $\rho \in A_{*}^{+}$and $x, y \in X$, $\rho([P(\cdot) x, y])$ is a regular measure on $\hat{G}$.

Take $\rho \in A_{*}^{+}$and define a semi-inner product on $X$ by $(x, y)_{\rho}=\rho([x, y]), x$, $y \in X$. Put $N_{\rho}=\left\{x \in X ;(x, x)_{\rho}=0\right\}$ and define $X_{\rho}$ to be the completion of the quotient space $X / N_{\rho}$ w.r.t. $(\cdot, \cdot)_{\rho}$. Then $X_{\rho}$ is a Hilbert space where we denote the inner product and the norm by $(\cdot, \cdot)_{\rho}$ and $\|\cdot\|_{\rho}$, respectively. Write $x_{\rho}=x$ $+N_{\rho} \in X / N_{\rho}$ for $x \in X$. Note that the inequality $\left\|x_{\rho}\right\|_{\rho} \leqq\|\rho\|^{1 / 2} \cdot\|x\|_{X}$ holds for $x \in X$. Let $s \rightarrow U(s)$ be a Gramian $\sigma$-weakly continuous GUR of $G$ on $X$. For each $s \in G$ define an operator $U_{\rho}(s)$ on $X / N_{\rho}$ by $U_{\rho}(s) x_{\rho}=(U(s) x)_{\rho}, x \in X$. Then $U_{\rho}(s)$ is well-defined, maps $X / N_{\rho}$ onto itself and is isometry on $X / N_{\rho}$. Hence $U_{\rho}(s)$ can be uniquely extended to a unitary operator, still denoted by $U_{\rho}(s)$, on $X_{\rho}$. Moreover, $s \rightarrow U_{\rho}(s)$ is a weakly continuous unitary representation of $G$ on the Hilbert space $X_{\rho}$ by the Gramian $\sigma$-weak continuity of $s \rightarrow U(s)$. By Stone's theorem there is a regular spectral measure $P_{\rho}$ on $\hat{G}$ such that $U_{\rho}(s)=$ $\int_{\hat{G}} \overline{\langle s, \chi\rangle} P_{\rho}(d \chi), s \in G$ where $\langle\cdot, \cdot\rangle$ is the duality pair of $G$ and $\hat{G}$ (cf. [18, Theorem 7.1$]$ ).

Now let $x, y \in X$ and $B \in \mathfrak{B}_{\hat{G}}$ be fixed and consider the functional $\Lambda$ on $A_{*}^{+}$ defined by

$$
\Lambda(\rho)=\left(P_{\rho}(B) x_{\rho}, y_{\rho}\right)_{\rho}, \quad \rho \in A_{*}^{+} .
$$

We first show that $\Lambda$ can be uniquely extended to a bounded linear functional on $A_{*}$.

5.2. Lemma. The functional $\Lambda$ on $A_{*}^{+}$defined by (5.1) is uniquely extended to a bounded linear functional on $A_{*}$.

Proof. It suffices to prove that if $\rho_{1}, \cdots, \rho_{n} \in A_{*}^{+}$and complex numbers $\alpha_{1}, \cdots, \alpha_{n}$ are such that $\sum_{j=1}^{n} \alpha_{j} \rho_{j}=0$, then $\sum_{j=1}^{n} \alpha_{j} \Lambda\left(\rho_{j}\right)=0$. Put $m_{j}(\cdot)=\left(P_{\rho_{j}}(\cdot) x_{\rho_{j}}\right.$, $\left.y_{\rho_{j}}\right)_{\rho_{j}}, 1 \leqq j \leqq n$ and define $m=\left|m_{1}\right|+\cdots+\left|m_{n}\right|$ where $\left|m_{j}\right|$ is the variation of $m_{\jmath}$. Then $m$ is a finite positive regular measure on $\hat{G}$ and the linear span of $G$, regarded as the dual group of $\hat{G}$, is dense in $L^{1}(\hat{G}, m)$. It follows that for any $\varepsilon>0$ there exist some $s_{1}, \cdots, s_{l} \in G$ and complex numbers $\beta_{1}, \cdots, \beta_{l}$ such that 


$$
\int_{\hat{G}}\left|1_{B}(\chi)-\sum_{k=1}^{l} \beta_{k} \overline{\left\langle s_{k}, \chi\right\rangle}\right| m(d \chi)<\frac{\varepsilon}{n}\left(\max _{1 \leqq j \leqq n}\left|\alpha_{j}\right|\right)^{-1}
$$

where $1_{B}$ is the characteristic function of $B$. Hence we have

$$
\begin{aligned}
& \left|\sum_{j} \alpha_{\jmath} \Lambda\left(\rho_{j}\right)-\sum_{j} \alpha_{j} \int_{\hat{G}} \sum_{k} \beta_{k} \overline{\left\langle s_{k}, \chi\right\rangle} m_{j}(d \chi)\right| \\
& \leqq \sum_{j}\left|\alpha_{j} \int_{\hat{G}}\left\{1_{B}(\chi)-\sum_{k} \beta_{k} \overline{\left\langle s_{k}, \chi\right\rangle}\right\} m_{\jmath}(d \chi)\right| \\
& \leqq \sum_{j}\left|\alpha_{j}\right| \int_{\hat{G}}\left|1_{B}(\chi)-\sum_{k} \beta_{k} \overline{\left\langle s_{k}, \chi\right\rangle}\right| m(d \chi)<\varepsilon .
\end{aligned}
$$

On the other hand, it follows from the assumption that

$$
\begin{aligned}
& \sum_{\jmath} \alpha_{j} \int_{\hat{G}} \sum_{k} \beta_{k} \overline{\left\langle s_{k}, \chi\right\rangle} m_{\jmath}(d \chi)=\sum_{\jmath} \alpha_{\jmath} \sum_{k} \beta_{k}\left(U_{\rho_{j}}\left(s_{k}\right) x_{\rho_{j}}, y_{\rho_{j}}\right)_{\rho_{j}} \\
& \quad=\sum_{\jmath} \alpha_{\jmath} \sum_{k} \beta_{k} \rho_{j}\left(\left[U\left(s_{k}\right) x, y\right]\right)=\sum_{\jmath} \alpha_{j} \rho_{j}\left(\sum_{k} \beta_{k}\left[U\left(s_{k}\right) x, y\right]\right)=0 .
\end{aligned}
$$

Consequently, $\left|\sum \alpha_{\jmath} \Lambda\left(\rho_{j}\right)\right|<\varepsilon$. Since $\varepsilon$ is arbitrary, we have $\sum \alpha_{\jmath} \Lambda\left(\rho_{j}\right)=0$, as desired. The boundedness of $\Lambda$ on $A_{*}$ is easily verified.

It follows from 5.2. Lemma that there is a unique element $P_{x, y}(B) \in A$ such that $\Lambda(\theta)=\theta\left(P_{x, y}(B)\right), \theta \in A_{*}$ and, in particular, $\left(P_{\rho}(B) x_{\rho}, y_{\rho}\right)_{\rho}=\rho\left(P_{x, y}(B)\right), \rho \in$ $A_{*}^{+}$. If $B$ varies over $\mathfrak{B}_{\hat{G}}$, the function $P_{x, y}(\cdot)$ defines an $A$-valued $\sigma$-weakly CA measure on $\hat{G}$. Then we have the following.

5.3. LEMMA. (1) For each $x, y \in X$ the relation

$$
[U(s) x, y]=\int_{\hat{G}} \overline{\langle s, \chi\rangle} P_{x, y}(d \chi), \quad s \in G
$$

holds where the integral is in the $\sigma$-weak topology of $A$.

(2) For each $x, y, z \in X$ and $a \in A$ the equalities $P_{a \cdot x, y}(\cdot)=a P_{x, y}(\cdot), P_{x+y, z}(\cdot)$ $=P_{x, z}(\cdot)+P_{y, z}(\cdot)$ and $P_{x, y}(\cdot)=P_{y, x}(\cdot)^{*}$ hold.

(3) For each $B \in \mathfrak{B}_{\hat{G}}$ and $y \in X$ the function $x \rightarrow P_{x, y}(B)$ from $X$ into $A$ is a bounded module map, i.e., $P_{., y}(B) \in X^{*}$.

Proof. (1) Let $x, y \in X$. For every $\rho \in A_{*}$ it holds that

$$
\begin{aligned}
\rho([U(s) x, y]) & =\left(U_{\rho}(s) x_{\rho}, y_{\rho}\right)_{\rho}=\int_{\hat{G}} \overline{\langle s, \chi\rangle}\left(P_{\rho}(d \chi) x_{\rho}, y_{\rho}\right)_{\rho} \\
& =\int_{\hat{G}} \overline{\langle s, \chi\rangle} \rho\left(P_{x, y}(d \chi)\right)=\rho\left(\int_{\hat{G}} \overline{\langle s, \chi\rangle} P_{x, y}(d \chi)\right) .
\end{aligned}
$$

This is enough to prove (5.2).

(2) Let $x, y \in X$ and $a \in A$, and take $\rho \in A_{*}^{+}$. By $[U(s)(a \cdot x), y]=a[U(s) x, y]$ 
we have $\int_{\hat{G}} \overline{\langle s, \chi\rangle} \rho\left(P_{a \cdot x, y}(d \chi)\right)=\int_{\hat{G}} \overline{\langle s, \chi\rangle} \rho\left(a P_{x, y}(d \chi)\right)$ for $s \in G$. Since $\rho\left(P_{a \cdot x, y}(\cdot)\right)$ and $\rho\left(a P_{x, y}(\cdot)\right)$ are regular, they coincide. This is enough to show that $P_{a \cdot x, y}(\cdot)$ $=a P_{x, y}(\cdot)$. Other equalities can be checked in a similar manner.

(3) Let $B \in \mathfrak{B}_{\hat{G}}$ and $y \in X$. It follows from (2) that $x \rightarrow P_{x, y}(B)$ is a module map. To see the boundedness, let $\rho \in A_{*}^{+}$. Then we have that $\left|\rho\left(P_{x, y}(B)\right)\right|=$ $\left|\left(P_{\rho}(B) x_{\rho}, y_{\rho}\right)_{\rho}\right| \leqq\left\|x_{\rho}\right\|_{\rho} \cdot\left\|y_{\rho}\right\|_{\rho} \leqq\|\rho\| \cdot\|x\|_{X} \cdot\|y\|_{X}$ for $x \in X$. Thus $\left\|P_{x, y}(B)\right\| \leqq$ $4\|x\|_{X} \cdot\|y\|_{X}, x \in X$. Therefore $P \cdot, y(B)$ is bounded.

Assume that $X$ is self-dual. Then it follows from 5.3. Lemma (3) that for each $y \in X$ and $B \in \mathfrak{B}_{\hat{G}}$ there is a unique $z \in X$ such that $P_{x, y}(B)=[x, z], x \in X$. Define $z=P(B) y$. Then $P(B)$ is a well-defined operator in $B(X)$ and $P(\cdot)$ is a $B(X)$-valued Gramian $\sigma$-weakly CA measure on $\hat{G}$ such that $U(s)=\int_{\hat{G}} \overline{\langle s, \chi\rangle} P(d \chi)$, $s \in G$ where the integral is in the Gramian $\sigma$-weak topology. All we have to do is to show that $P(\cdot)$ is a Gramian spectral measure.

\subsection{Lemma. $P(\cdot)$ is a Gramian spectral measure on $\hat{G}$.}

Proof. Let $B \in \mathfrak{B}_{\hat{G}}$ be fixed. It follows from 5.3. Lemma (2) that $[x, P(B) y]$ $=P_{x, y}(B)=P_{y, x}(B)^{*}=[y, P(B) x]^{*}=[P(B) x, y]$ for $x, y \in X$. Hence $P(B) \in \mathfrak{A}(X)$ with $P(B)^{*}=P(B)$. Now we show that $P(B)^{2}=P(B)$. First we see that $\left(x_{\rho},(P(B) y)_{\rho}\right)_{\rho}=\rho([x, P(B) y])=\rho\left(P_{x, y}(B)\right)=\left(x_{\rho}, P_{\rho}(B) y_{\rho}\right)_{\rho}$ for $x, y \in X$ and $\rho \in$ $A_{*}^{+}$. Hence $(P(B) y)_{\rho}=P_{\rho}(B) y_{\rho}, y \in X, \rho \in A_{*}^{+}$. Consequently we have $(P(B) y)_{\rho}$ $=P_{\rho}(B)^{2} y_{\rho}=P_{\rho}(B)\left(P_{\rho}(B) y_{\rho}\right)=P_{\rho}(B)(P(B) y)_{\rho}=\left(P(B)^{2} y\right)_{\rho}$ for $y \in X$ and $\rho \in A_{*}^{*}$. Therefore $P(B)^{2}=P(B)$, as desired. It is clear that $\rho([P(\cdot) x, y])$ is a regular measure on $\hat{G}$ for each $x, y \in X$ and $\rho \in A_{*}^{+}$. Thus $P(\cdot)$ is a Gramian spectral measure.

We summarize these discussions in the following theorem.

5.5. Theorem. Let $X$ be a self-dual Hilbert A-module and $s \rightarrow U(s)$ be a Graman $\sigma$-weakly contınuous GUR of $G$ on $X$. Then there is a Gramian spectral measure $P$ on $\hat{G}$ such that

$$
U(s)=\int_{\hat{G}} \overline{\langle s, \chi\rangle} P(d \chi), \quad s \in G
$$

where the integral is in the Graman $\sigma$-weak topology.

Now we can prove a Bochner type theorem as follows.

5.6. Corollary. For an A-valued weakly continuous $P D$ function $\Gamma$ on $G$ there is an A-valued $\sigma$-weakly $C A$ measure $F$ on $\hat{G}$ such that

$$
\Gamma(s)=\int_{\hat{G}} \overline{\langle s, \chi\rangle} F(d \chi), \quad s \in G
$$


where the integral is in the $\sigma$-waak topology of $A$.

Proof. It follows from 4.2. Proposition that there exist a Hilbert $A$-module $X_{\Gamma}$, a GUR $s \rightarrow U_{0}(s)$ of $G$ on $X_{\Gamma}$ and a cyclic vector $x_{0} \in X_{\Gamma}$ such that $\Gamma(s)$ $=\left[U_{0}(s) x_{0}, x_{0}\right]_{\Gamma}, s \in G$ where $[\cdot, \cdot]_{\Gamma}$ is the Gramian on $X_{\Gamma}$. Again by 4.2. Proposition $\Gamma$ is $\sigma$-weakly continuous since weak and $\sigma$-weak topologies coincide on bounded subsets of $A$. Hence we can see that $s \rightarrow U_{0}(s)$ is Gramian $\sigma$-weakly continuous. Then $s \rightarrow U_{0}(s)$ can be uniquely extended to a Gramian $\sigma$-weakly continuous GUR $s \rightarrow U(s)$ of $G$ on the self-dual Hilbert $A$-module $X_{\Gamma}^{*}$. Consequently, by 5.5. Theorem, there is a Gramian spectral measure $P$ on $\hat{G}$ such that $U(s)=\int_{\hat{G}} \overline{\langle s, \chi\rangle} P(d \chi), s \in G$. Putting $F(\cdot)=\left[P(\cdot) x_{0}, x_{0}\right]$ where $[\cdot, \cdot]$ is the Gramian on $X_{\Gamma}^{*}$, we have that $F$ is an $A$-valued $\sigma$-weakly CA measure on $\hat{G}$ and that, for $s \in G$,

$$
\begin{aligned}
\Gamma(s) & =\left[U(s) x_{0}, x_{0}\right]=\left[\int_{\hat{G}} \overline{\langle s, \chi\rangle} P(d \chi) x_{0}, x_{0}\right]=\int_{\hat{G}} \overline{\langle s, \chi\rangle}\left[P(d \chi) x_{0}, x_{0}\right] \\
& =\int_{\hat{G}} \overline{\langle s, \chi\rangle} F(d \chi) .
\end{aligned}
$$

\section{Hilbert $A$-module valued processes.}

Let $G$ be a locally compact abelian group and $X$ be a Hilbert $A$-module. We consider $X$-valued processes over $G$.

6.1. Definition. (1) An $X$-valued process $\{x(t)\}$ over $G$ is a map $t \rightarrow x(t)$ from $G$ into $X$.

(2) The covariance function $\Gamma$ of a process $\{x(t)\}$ is defined by $\Gamma(s, t)=$ $[x(s), x(t)], s, t \in G . \quad \Gamma$ is an $A$-valued PDK on $G \times G$.

(3) A process $\{x(t)\}$ is said to be stationary if its covariance function $\Gamma(s, t)$ depends only on $s t^{-1}$ and, putting $\Gamma(s, t)=\Gamma\left(s t^{-1}\right)$, if $\Gamma$ is an $A$-valued weakly continuous function on $G$.

(4) For a process $\tilde{x}=\{x(t)\}$ the time domain $\mathfrak{S}(\tilde{x})$ and an observation space $\mathfrak{S}(\tilde{x} ; D)$ of a subset $D$ of $G$ are defined as submodules by $\mathfrak{S}(\tilde{x})=\mathfrak{S}\{x(t) ; t \in G\}$

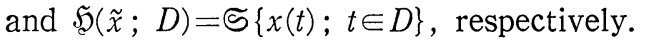

(5) Let $\tilde{x}=\{x(t)\}$ be an $X$-valued process and $\tilde{y}=\{y(t)\}$ be a $Y$-valued process, $Y$ being a Hilbert $A$-module. Then $\tilde{x}$ and $\tilde{y}$ are said to be equivalent if there exists a Gramian unitary operator $U: \mathfrak{S}(\tilde{x}) \rightarrow \mathfrak{H}(\tilde{y})$ such that $U x(t)=y(t)$, $t \in G$.

Then the following is easily proved.

6.2. Proposition. (1) For any A-valued $P D K \Gamma$ on $G \times G$ there is some Hilbert A-module valued process with the covariance function $\Gamma$. 
(2) Let $\tilde{x}$ be an $X$-valued process with the covariance function $\Gamma$. Then we have, for each subset $D$ of $G, \mathfrak{S}(\tilde{x} ; D) \cong D \otimes_{\Gamma} A$ and, in partıcular, $\mathfrak{S}(\tilde{x}) \cong G \otimes_{\Gamma} A$ where $D \otimes_{\Gamma} A$ was constructed in section 3 .

(3) Let $\tilde{x}$ be an $X$-valued process and $\tilde{y}$ be a $Y$-valued process, $Y$ being a Hilbert A-module. Then $\tilde{x}$ and $\tilde{y}$ are equivalent if and only if their covariance functions are identical.

(4) Stationarity is invariant within equivalence. More precisely, let $\tilde{x}$ and $\tilde{y}$ be as in (3) above. If they are equivalent and $\tilde{x}$ is stationary, then $\tilde{y}$ is also stationary.

(5) Let $\{x(t)\}$ be an X-valued statıonary process with the covariance function

$\Gamma$. Then there exist an $X^{*}$-valued $C A$ orthogonally scattered measure $\xi$ and an $A$-valued $C A$ measure $F$ on $\hat{G}$ such that

$$
x(t)=\int_{\hat{G}} \overline{\langle t, \chi\rangle \xi}(d \chi), \quad \Gamma(t)=\int_{\hat{G}} \overline{\langle t, \chi\rangle} F(d \chi), \quad t \in G
$$

where the orthogonal scatteredness of $\xi$ means that $[\xi(A), \xi(B)]=0$ for every disjoint pair $A, B \in \mathfrak{B}_{\hat{G}}$.

Let $(\Omega, \mathfrak{B}, \mu)$ be a probability measure space and $E$ be a Banach space with the dual space $E^{*}$. An $E$-valued function $x$ on $\Omega$ is said to be of weak second order if it is weakly measurable and $f^{*}(x(\cdot)) \in L^{2}(\Omega, \mu)$ for every $f^{*} \in E^{*}$. For each such function $x$ there is an operator $T_{x}: E^{*} \rightarrow L^{2}(\Omega, \mu)$ such that $\left(T_{x} f^{*}\right)(\cdot)$ $=f^{*}(x(\cdot)), f^{*} \in E^{*}$. If $E$ is separable, then $T_{x}^{*}: L^{2}(\Omega, \mu) \rightarrow E \subset E^{* *}$ (cf. [19, 2.2. Proposition]). Putting $H=L^{2}(\Omega, \mu)$ and $L=E^{*}$, we define an $E$-valued process over $G$ of weak second order to be a $B(L, H)$-valued process over $G$ where $B(L, H)$ is the Banach space of all bounded linear operators from $L$ into $H$. The case where $L$ is a Hilbert space was studied by Gangolli [4]. In this case $B(L, H)$ is a (right) Hilbert $B(L)$-module as was noted by Gangolli. Susiu and Valsescu [16] considered in this view point (see also Saworotnow [15]). The case where $L$ is an arbitrary Banach space was studied by several authors such as Cobanjan and Weron [2], Weron [19] and Miamee [8] (cf. [9]).

Let $\{x(t)\}$ be an $E$-valued process of weak second order, i.e., $\{x(t)\}$ is a $B\left(E^{*}, H\right)$-valued process. When $E$ is separable or reflexive, the adjoint process $\left\{x(t)^{*}\right\}$, which is $B\left(H, E^{* *}\right)$-valued, becomes a $B(H, E)$-valued process. The space $B(H, E)$ is a (right) Hilbert $B(H)$-module if we define a module action and a Gramian by $x \cdot a=x a$ and $[x, y]=y^{*} x$ for $x, y \in B(H, E)$ and $a \in B(H)$, respectively. Hence our theory is available in this respect.

\section{REFERENCES}

[1] Aronszajn, N., Theory of reproducing kernels, Trans. Amer. Math. Soc. 68 (1950), 337-404.

[2] Cobanjan, S. A. and Weron, A., Banach space valued stationary processes and 
their linear prediction, Dissertationes Math. 125 (1975), 1-45.

[3] Dixmier, J., C*-algebras, North-Holland, Amsterdam, 1977.

[4] Gangolli, R., Wide-sense stationary sequences of distributions on Hilbert space and the factorization of operator valued functions, J. Math. Mech. 12 (1963), 893-910.

[5] Iтон, S., A note on dilations in modules over $C^{*}$-algebras, J. London Math. Soc. (2) 22 (1980), 117-126.

[6] Kakihara, Y. ANd Terasaki, T., Hilbert $B(H)$-modules with applications I, Res. Rep. Inst. Inf. Sci. Tech. (Tokyo Denki Univ.) 5 (1979), 23-32.

[7] Kaplansky, I., Modules over operator algebras, Amer. J. Math. 75 (1953), 839858.

[8] Miamee, A.G., On $B(X, K)$-valued statıonary stochastıc processes, Indiana Univ. Math. J. 25 (1976), 921-932.

[9] Miamee, A.G. And Salehi, H., On the square root of a positive $B\left(3,3^{*}\right)$-valued function, J. Multivar. Anal. 7 (1977), 535-550.

[10] Nakamura, M. and Umegaki, H., A remark on theorems of Stone and Bochner, Proc. Japan Acad. 27 (1951), 506-507.

[11] Ozawa, M., Hilbert $B(H)$-modules and statıonary processes, Kodai Math. J. 3 (1980), 26-39.

[12] Paschke, W.L., Inner product modules over $B^{*}$-algebras, Trans. Amer. Math. Soc. 182 (1973), 443-468.

[13] RiefFel, M. A., Induced representations of $C^{*}$-algebras, Advances Math. 13 (1974), $176-257$.

[14] Saworotnow, P.P., A generalized Hilbert space, Duke Math. J. 35 (1968), 191197.

[15] Saworotnow, P.P., Abstract stationary processes, Proc. Amer. Math. Soc. 40 (1973), 585-589.

[16] Susiu, I. And Valsescu, I., A linear filtering problem in complete correlated actions, J. Multivar. Anal. 9 (1979), 599-613.

[17] Umegaki, H., Positive definite function and direct product Hilbert space, Tôhoku Math. J. 7 (1955), 206-211.

[18] Umegaki, H., An operator method for harmonic analysis on locally compact abelian group, MRC Tech. Summary Rep. \#725, Madison, Wisconsin, 1967.

[19] Weron, A., Prediction theory in Banach space, Lecture Notes in Math. No. 472, pp. 207-228, Springer-Verlag, New York, 1975.

Department of Mathematical Science

TOKYO DENKI UNIVERSITY

IsHizaKa, HatoYAMA-MACHI, HiKI-GUN

SAITAMA-KEN 350-03, JAPAN 